Station 9.



Station 10 (im östlichen Thurme der Wasserkunst).

Station 9
Reductions-Elemente $\left\{\begin{array}{l}\text { Station } 9 \\ \mathrm{R}=0^{\mathrm{T}} 7055\end{array}-\right.$ Nördlicher Domthurm

Anmerkungen: Eingestellt sind sümmtliche eisernen Stangen an den Thurmknöpfen oben.

Die Winkel sind mit einem 8zölligen Theodoliten von Ertel genessen.

Aus den vorhergehenden Messungen sind die folgenden Coordinaten abgeleitet:

\begin{tabular}{|c|c|c|c|c|c|}
\hline & $x$ & & & $x$ & $y$ \\
\hline Nördlicher Marienthurm & $0^{\mathrm{T}} 000$ & $0^{\mathrm{T}} 000$ & Petri Kirchthurm & $+120,822$ & $+51,228$ \\
\hline Südlicher Marieuthurm & $+14,791$ & $+2,405$ & Mittlerer Rathhausthurm & $+57,123$ & $-31,035$ \\
\hline Station 1 & $+606,698$ & $-725,638$ & Catharinen Kirchthurm & $-68,435$ & $-177,994$ \\
\hline Station 2 & $+430,283$ & $-759,085$ & Jacobi Kirchthurm & $-168,071$ & $-139,200$ \\
\hline Station 3 & $+308,042$ & $-782,259$ & Heil. Geist Kirchthurm & $-190,580$ & $-181,784$ \\
\hline Station 4 & $-235,798$ & $-885,360$ & Burg-Thurm & $-338,630$ & $-225,698$ \\
\hline Station 5 & $+552,347$ & $-530,338$ & Stange der Navigations-Schule & $+567,089$ & $-121,880$ \\
\hline Station 6 & $+568,638$ & $-135,656$ & Ecke 1 der Navig.-Schule & $+567,246$ & $-126,010$ \\
\hline Station 7 & $+555,014$ & $-125,110$ & Ecke $2 ", "$ & $+563,580$ & $-120,931$ \\
\hline Station 8 & $+527,668$ & $-147,073$ & Ecke 3 ", & $+569,498$ & $-114,915$ \\
\hline Station 9 & $+552,710$ & $-90,404$ & Ecke $4 "$ & $+574,059$ & $-119,193$ \\
\hline Nördlicher Domthurm & $+399,8 i 6$ & $-12,771$ & Ort des Passageninstruments 1831 & $+563,430$ & $-132,678$ \\
\hline Süllicher Jomthurm & $+412,956$ & $-12,573$ & Ort des Passageninstruments 1833 & $+571,104$ & $-135,657$ \\
\hline Anneu Kirchthurm & $+282,188$ & $-151,766$ & \multirow{2}{*}{\multicolumn{3}{|c|}{$+x$ bedeutet $x$ Toisen südlich, }} \\
\hline Aegidien Kirchthurm & $+231,414$ & $-167,061$ & & & \\
\hline Stange eines hohen Giebels in der & & & $-x$ & nördlich, & \\
\hline Krähenstrasse & $+208,873$ & $-234,411$ & $+y$ & westlich, & \\
\hline Westl. Thurm der Wasserkunst & $+189,318$ & $-310,602$ & $-y$ & östlich. & \\
\hline
\end{tabular}

Oestl. Thurm der Wasserkunst $\quad+187,700 \quad-324,756$

Nyegaard.

\title{
Beobachtung der Mondfinsterniss am 13. October 1856 auf der Hamburger Sternwarte, von Herrn G. Rümker.
}

Von der neulichen Mondfinsterniss habe ich folgende Beobachtungen am hiesigen Refractor erhalten. - Des schlecht begränzten Kernschattens wegen sind die einzelnen Momente wohl über 10" unsicher. 


\begin{tabular}{|c|c|c|c|c|}
\hline \multirow[b]{2}{*}{ Anfang } & \multicolumn{2}{|c|}{ Eintritte } & \multicolumn{2}{|c|}{ Austritte } \\
\hline & $10^{\mathrm{h}}$ & $1^{\mathrm{m}}$ & & \\
\hline Grimaldi Mitte & 10 & $421^{\prime}$ & $12^{\mathrm{h}} 13^{\mathrm{n}}$ & ${ }^{\mathrm{n}} 10^{\mathrm{s}}$ \\
\hline Gassendi Mitte & 101 & 1042 & 1225 & 37 \\
\hline Rainer? & 10 & 1047 & & \\
\hline Marius? & 10 & 1552 & & \\
\hline Keppler Mitte & 10 & 1734 & 1215 & 1 \\
\hline Ptolomäus & $10:$ & 207 & & \\
\hline Tycho 1 & 101 & 1943 & & \\
\hline " Mitte & 102 & 2021 & & \\
\hline,$\quad 11$ & 102 & 2055 & & \\
\hline Mayer & 102 & 2151 & & \\
\hline Aristarch Mitte & 102 & 230 & 123 & 55 \\
\hline Copernicus 1 & 102 & $26 \quad 9$ & 1219 & 11 \\
\hline Mitte & $10:$ & 2650 & 1220 & 20 \\
\hline$n \quad$ II & 102 & 2744 & 1221 & 0 \\
\hline Pytheas & $10:$ & 328 & 1214 & 26 \\
\hline Manilius Mitte & 10 & $44 \quad 38$ & & \\
\hline Archinnedes 1 & 10 & $\begin{array}{ll}44 \quad 8 \\
\end{array}$ & & \\
\hline " Mitte & 10 & $44 \quad 56$ & & \\
\hline 11 & 10 & 4550 & & \\
\hline Menelaus Mitte & 10 & $48 \quad 45$ & 1234 & 8 \\
\hline Godenius & 12 & 5220 & & \\
\hline Plato I & 10 & $56 \quad 14$ & 1156 & 43 \\
\hline " Mitte & 10 & 5T 14 & 1157 & 52 \\
\hline , II & 10 & $57 \quad 59$ & 1158 & 44 \\
\hline
\end{tabular}

\begin{tabular}{|c|c|c|c|c|}
\hline & & ntritte & & Austritte \\
\hline Eudoxus Mitte & $11^{\mathrm{h}}$ & $3^{m} 21^{\prime}$ & & $12^{\mathrm{h}} 10^{\mathrm{m}} 5^{\mathrm{s}}$ \\
\hline Proclus & 11 & 359 & & \\
\hline Mare Crisium I & 11 & 418 & & \\
\hline$" \quad, \quad$ Mitte & 11 & 657 & & \\
\hline$" \quad$ II & 11 & 959 & & \\
\hline Endymion Mitte & $11:$ & 2130 & I.R. & $12 \quad 535$ \\
\hline Total? & $11:$ & 33 & & \\
\hline
\end{tabular}

Bald nach Mitternacht begann der Himmel, der bis dahin ganz heiter gewesen war, sich zu trüben, so dass wir hier das Ende der Finsterniss nicht sehen konnten.

Meiner Ansicht nach war sie, wenn nicht total, der Totalität so nahe, dass sich unmöglich mit Sicherheit behaupten lässt, ein bestimmter Theil des Mondes sei im Verlauf derselben vom Erdschatten frei geblieben. Hier war im $5 \frac{1}{2}$ füssigen Refractor um $11^{\mathrm{h}} 33^{\mathrm{m}}$ der ganze Mond vom Schatten bedeckt, und nur der N.W.-Rand leuchtete ein weniges gelblicher als der übrige Theil.

Mit blossem Auge war freilich noch immer ein ziemlicher Theil des Mondes in dunkelgelbem Lichte zu sehen; wogegen der übrige Theil, vielleicht des Contrastes halber, eine ungewobnlich trübe schwärzlich rothe Färbung erhielt.

Sternwarte Hamburg 1856. Nov. 24.

George Riimker.

\section{Aus einem Schreiben des Herrn Dr. Lehmann an den Herausgeber.}

In Folge eines kleinen Rechnungsfehlers von meiner Seite haben sich in $\mathcal{N}_{2} 1049$ und 1050 folgende Versehen eingeschlichen, um deren gelegentliche Anzeige ich bitte.

J6 1049 S. 268 in der sechstletzten Zeile des 37sten $\$$ statt ununterbrochen lies fast ununterbrochen.

In der folgenden Zuile statt 0,00006 lies 0,00005.

No 1050 S. 278 in der letzten Tabelle in Intervall $x=0,45$ his $x=0,60$ setze man +2 statt $+2,5$, im Intervall $x=0,60$ bis $x=0,75$ aber +1 statt $+1,5$.
S. 279, 280 in der Tabelle gehört zu $x=0,60$ nicht $\frac{d y}{d x}=-0,04887$, sondern $-0,04886$, und die rechts daneben stehenden Differebzen sind in -147 und $-157 \mathrm{zu}$ verwandeln.

S. 280 in derselben Tabelle in dem von $x=0,45$ bis $x=0,60$ reichenden Intervall ist $\log \frac{r}{a}=$ 9,696171. statt 9,696172. zu setzen.

S. 280 Z. 15 v. u. statt 9,185779 . lies 9,185778 .

Potsdam, den 18. Nov. 1856.
W. Lelimann.

\section{I n h a $\mathbf{l}$ t.}

(Zu Nr. 1057.) Polhōho von Lübeck, abgeleitet aus Beobachtungen an einem tragbaren Repsold'schen Passageninstrumente im ersten Verticale 1. Messunget in und bei Lübeck im September 1833, ron Capitain Nyegaurd 7. -

Beobachtung der Mondfinsterniss am 13. October 1856, von Herrn G. Rumker 13. -

Auw einem Schreiben des Herrn Dr. Lehmann an den Horausgeber 15. - 